\title{
Structure-function analyses of the HTLV-I Rex and HIV-1 Rev RNA response elements: insights into the mechanism of Rex and Rev action
}

\author{
Yasmath F. Ahmed, Sarah M. Hanly, Michael H. Malim, Bryan R. Cullen, and Warner C. Greene' \\ Howard Hughes Medical Institute, Department of Medicine and Department of Microbiology and Immunology, Duke \\ University Medical Center, Durham, North Carolina 27710 USA
}

\begin{abstract}
The ability of the Rex protein of the type I human T-cell leukemia virus (HTLV-I) to regulate expression of the retroviral gag and env structural genes post-transcriptionally is critically dependent on the presence of a Rex response element (RexRE). This cis-regulatory sequence is located within the retroviral 3' long terminal repeat and coincides with a predicted, highly stable RNA stem-loop structure. Rex action requires both the overall secondary structure intrinsic to the RexRE and specific sequences from one small subregion of this large structure. This small subregion likely forms a protein-binding site for Rex or a cellular RNA-binding factor. Whereas Rex can functionally replace the Rev protein of the type 1 human immunodeficiency virus (HIV-1) through its interaction with the analogous Rev response element (RevRE), distinct subregions of this HIV-1 RNA element mediate the responses to Rex and Rev. Strikingly, Rex acts as a dominant repressor of Rev action, following the deletion of the Rex responsive subregion of the RevRE. Similarly, Rev inhibits Rex function in a dominant manner when the Rev responsive subregion of the RevRE is deleted. Together, these findings suggest that Rex and Rev not only interact with their respective RNA response elements but also may either form mixed inactive multimers or interact with a common cellular factor(s). If binding of a common host protein is involved, this factor likely plays a central role either in spliceosome assembly or nuclear RNA transport.
\end{abstract}

[Key Words: HTLV-I; HIV-1; RNA transport; RNA splicing; RNA secondary structure; polyadenylation]

Received January 31, 1990; revised version accepted April 9, 1990.

The type I human T-cell leukemia virus (HTLV-I) and the type 1 human immunodeficiency virus (HIV-1), though only distantly related and etiologically associated with very different pathological processes, both require the translation of two incompletely spliced mRNA species for production of their gag and env structural gene products (Seiki et al. 1985; Yoshida and Seiki 1987; Cullen and Greene 1989). Both of these viruses encode a trans-acting protein, Rex in HTLV-I and Rev in HIV-1, which plays an essential role in regulating gag and env gene expression (Inoue et al. 1987; Hidaka et al. 1988; Malim et al. 1988, 1989b; Sadaie et al. 1988; Terwilliger et al. 1988; Hanly et al. 1989). Specifically, Rex and Rev promote the cytoplasmic appearance of the incompletely spliced gag and env mRNAs, thereby allowing their translation and the assembly of infectious virions. In addition, both viruses contain distinct cis-regulatory sequences, termed the Rev and Rex response elements (RevRE and RexRE), that are coincident with predicted large and stable RNA stem-loop structures. The

'Corresponding author. sequence-specific action of Rex and Rev is dependent on the presence of these response elements (Rosen et al. 1988; Seiki et al. 1988; Emerman et al. 1989; Hadzopoulou-Cladaras et al. 1989; Hammarskjold et al. 1989; Hanly et al. 1989; Malim et al. 1989b|. Although neither the trans-activators nor their response elements exhibit significant homology, we have demonstrated previously that Rex is able to functionally replace the Rev protein, as evidenced by its rescue of the replication of Rev-deficient HIV-1 proviruses (Rimsky et al. 1988). The remarkably similar action and functional overlap of these viral trans-activators in the divergent HTLV and HIV suggests a partial pattern of convergent evolution within these human pathogens.

In this paper we further dissect the mechanism of Rex action, focusing on the structure and function of the RexRE and RevRE. We have shown previously that cisacting sequences within the $3^{\prime}$ long terminal repeat (LTR) of HTLV-I are sufficient to confer Rex responsiveness to a heterologous expression vector (Hanly et al. 1989|. Several observations have suggested that these sequences function at the RNA, rather than the DNA, level. First, the RexRE is coincident with a predicted 
highly ordered RNA stem-loop structure. Second, the element functions in an orientation-dependent, but position-independent, manner, provided it is represented in the primary RNA transcript. We now demonstrate that the function of the RexRE depends both on its general secondary structure and one small subregion that likely forms a protein-binding site. In terms of the RevRE, we demonstrate that Rex and Rev function through different subregions. Furthermore, we show that Rex acts as a dominant repressor of Rev when a deleted version of the RevRE lacking the Rex subregion is tested. Conversely, Rev serves as a dominant repressor of Rex when the Rev-binding site is deleted from the RevRE. Together, these findings suggest that Rex and Rev not only recognize their RNA target sequences but also may either form inactive mixed multimers or alternatively interface with a common cellular factor(s) that is perhaps involved in RNA splicing or RNA transport from the cell nucleus.

\section{Results}

Rex responsiveness is conferred to both homologous and heterologous viral expression vectors by addition of the RexRE

Recently, we have reported that sequences within the $3^{\prime}$ LTR of HTLV-I previously implicated in Rex responsiveness (Seiki et al. 1988) are also predicted to form a very stable and highly significant RNA stem-loop structure (Hanly et al. 1989). To determine whether these isolated sequences are sufficient to confer Rex responsiveness to an HTLV-I expression vector, we compared the activities of the plasmids pgTAX and pgTAX-R' (Fig. 1A). These vectors were designed to distinguish between the translation of spliced and unspliced HTLV-I mRNA species and, thus, to serve as indicators of Rex responsiveness. The pgTAX vector (Hanly et al. 1989) contains the two coding exons of the tax gene separated by an intron that coincides with the coding sequence of the env gene (Fig. 1A). The spliced mRNA derived from this vector encodes the $40-\mathrm{kD}$ Tax protein, whereas translation of the unspliced mRNA yields the $62-\mathrm{kD}$ Env precursor protein. However, as we have reported previously (Hanly et al. 1989), this plasmid is refractory to Rex action due to the absence of the RexRE. Thus, in the presence or absence of Rex, only the Tax protein is synthesized (Fig. $1 \mathrm{~A}$, lanes 3 and 4$)$.

We then inserted a DNA fragment spanning the predicted RNA stem-loop structure, containing 35 nucleotides of $5^{\prime}$-flanking sequences and 44 nucleotides of 3 '-flanking sequences, into the pgTAX vector immediately downstream of the tax gene, thus forming the pgTAX-R' plasmid (Fig. 1A). In the absence of Rex, the pgTAX-R' plasmid yielded primarily the $40-k D$ Tax protein and trace amounts of the 62-kD HTLV-I Env protein (Fig. 1A, lane 1). However, cotransfection of a Rex expression plasmid (pcREX; Rimsky et al. 1988) resulted in increased amounts of the $62-\mathrm{kD}$ Env protein, indicating enhanced expression of the unspliced mRNA species derived from this vector (Fig. 1A, lane 2). Con- comitant with the Rex-induced increase in env gene expression, the levels of Tax protein declined, indicating a shift from the translation of spliced to unspliced forms of vector mRNA. These results confirm the finding that sequences within the 3' LTR of HTLV-I, coincident with a predicted highly ordered RNA stem-loop structure, are sufficient to confer Rex responsiveness in an HTLV-I expression system.

Because the sequences within the RexRE have also been implicated in polyadenylation of viral transcripts (Seiki et al. 1983), we opted to perform all further analyses of the RexRE in a heterologous vector system, HIV-1 pgDTAT. This system permits a mutational analysis of the RexRE that is totally segregated from any role this element may serve in polyadenylation. Like pgTAX, the HIV-1 pgDTAT vector (Malim et al. 1989b) is designed to distinguish between the translation of spliced and unspliced mRNA species. The full-length HIV-1 Tat protein is 86 amino acids in length and represents the translation product of fully spliced vector mRNAs (Fig. 1B). In addition, unspliced transcripts from this vector encode a 72-amino-acid truncated version of Tat, reflecting the presence of residues derived from only the first tat exon. Thus, Rex action can be assayed by discrimination of the truncated 72-amino-acid polypeptide from the full-length 86 -amino-acid form of Tat. In the absence of a response element, all transcripts from $\mathrm{pg} \triangle \mathrm{TAT}$ are fully spliced and encode only the 86-aminoacid Tat, both in the presence and absence of Rex (Fig. $1 B$, lanes 1 and 2). In contrast, insertion of the RexRE in the tat gene intron, thus forming $\operatorname{pg} \Delta \mathrm{TAT} / \mathrm{R}^{\prime}$ (Fig. $1 \mathrm{~B}$ ), permits translation of unspliced mRNAs yielding the truncated Tat protein only in the presence of Rex (Fig. $1 B$, lanes 3 and 4 ). These results confirm the finding that the RexRE is sufficient to confer Rex responsiveness in a heterologous expression system independent of polyadenylation (Hanly et al. 1989).

\section{One subregion of the RexRE is required for Rex responsiveness}

To characterize the RexRE further, we employed its predicted secondary structure as a guide for the construction of serial internal deletion mutations (Fig. 2A). Both large and small mutations were introduced into the RexRE to assess whether the general secondary structure of this element is required for Rex responsiveness, as well as to determine whether any small subregions exist that are essential for Rex action. Each of the resultant mutant RexREs was inserted into the pgDTAT vector for analysis. Three large internal deletions within the RexRE, which drastically altered the predicted stability of this element, were prepared by either destroying the primary stem (deletion between nucleotides 317 and 370 ) or removing more than one of the stem-loop subregions (deletion between nucleotides 386 and 465, which removes three loops, and deletion between nucleotides 403 and 465, which removes two loops; Fig. 2A). Each of these large deletions produced a complete loss of Rex responsiveness, indicating that the general 

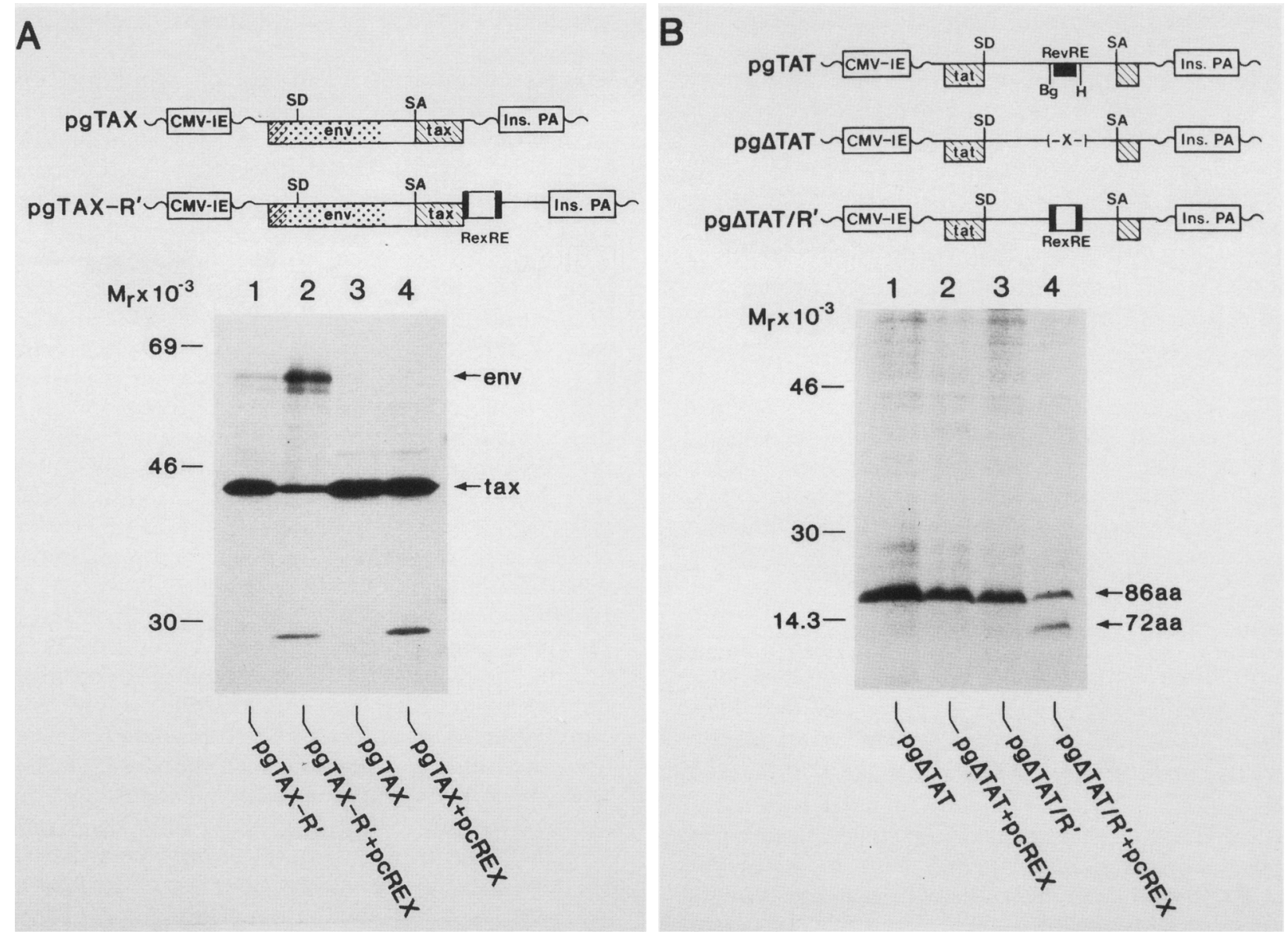

Figure 1. Immunoprecipitation analysis of Rex action mediated through the HTLV-I RexRE. $(A)$ Construction and phenotypic analysis of the pgTAX and pgTAX-R' HTLV-I viral expression vectors. Organization of HTLV-I genomic sequences, including the two tax gene exons (hatched pattern) and the overlapping env gene exon (stippled pattern), is depicted. The splice donor (SD) and splice acceptor $(\mathrm{SA})$ for the tax gene, as well as the cytomegalovirus immediate early promoter (CMV-IE) and rat preproinsulin gene polyadenylation site (Ins. PA) are indicated. The pgTAX- $\mathrm{R}^{\prime}$ plasmid is derived from pgTAX by insertion of the RexRE (map position 302-635) (Seiki et al. 1983) immediately downstream of the second tax exon. Monkey kidney COS cells were transfected with these plasmids either in the presence (lanes 2 and 4) or absence (lanes 1 and 3) of the Rex expression vector pcREX. These cells were cultured for $48 \mathrm{hr}$ and then biosynthetically labeled for $2 \mathrm{hr}$ with $\left[{ }^{35} \mathrm{~S}\right]$ cysteine. Cell lysates were immunoprecipitated with both the anti-Env $0.5 \alpha$ monoclonal antibody and anti-Tax antisera. Immunoprecipitated proteins were fractionated on $10 \%$ SDS-polyacrylamide gels. The $62-\mathrm{kD}$ Env and $40-\mathrm{kD}$ Tax proteins, as well as molecular weight standards, are indicated. Unexpected coprecipitation of the $27-\mathrm{kD}$ Rex protein, perhaps related to its overexpression, is seen in lanes 2 and 4. (B) Analysis of RexRE function in the heterologous HIV-1 expression vector $\mathrm{pg} \Delta T A T / R^{\prime}$. The pgTAT expression vector is derived from HIV-1 genomic sequences and includes both exons of the tat gene (Malim et al. 1988). pg $\triangle T A T$ is derived from pgTAT by deletion of sequences corresponding to the RevRE located between the indicated $B g I I I(B g)$ and HindIII $(\mathrm{H})$ restriction sites (Malim et al. 1989b). pg $\Delta$ TAT/R' is identical to pg $\Delta$ TAT, except for insertion of the RexRE in the tat gene intron, at the unique $X b a l$ restriction site (X). The splice donor (SD) and splice acceptor (SA) for the tat gene, as well as the cytomegalovirus immediate early promoter (CMV-IE) and rat preproinsulin gene polyadenylation site (Ins. PA) are indicated. These plasmids were transfected into COS cells in the presence (lanes 2 and 4) or absence (lanes 1 and 3) of the Rex expression vector pcREX. Cell lysates from biosynthetically labeled cells were immunoprecipitated with anti-Tat polyclonal antibodies. Immunoprecipitated proteins were fractionated on $14 \%$ SDS-polyacrylamide gels. The 86 - and 72 -amino-acid forms of the Tat protein (Malim et al. 1988), corresponding to the translation of spliced and unspliced mRNAs, respectively, are indicated.

secondary structure of this element is critical for its function (Fig. 2B, lanes 3-5). In contrast, the introduction of many smaller deletions within these individual stems or stem-loop structures in general did not alter Rex responsiveness. Specifically, REM $1(\Delta 317-342)$, REM 2 ( $\Delta 365-370)$, REM 3 ( $\Delta 377-399)$, REM 4 ( $\Delta 403-$ $420)$, and REM $5(\Delta 422-467)$ left the Rex response intact (Fig. 2C, lanes 2-6). In addition, several other mutations in distant regions, namely REM $6(\Delta 472-477)$, REM 7

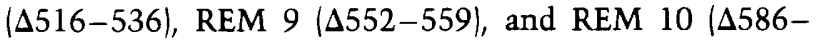
589 ) also did not alter the Rex response significantly. However, the REM 8 mutant, corresponding to deletion between bases 506 and 546, produced a nearly complete loss of Rex responsiveness (Fig. 2C, lane 9).

To define more precisely the importance of the REM 8 subregion of the RexRE, four additional substitution mutations were constructed in this portion of the element (Fig. 3A). The highlighted nucleotides corre- 

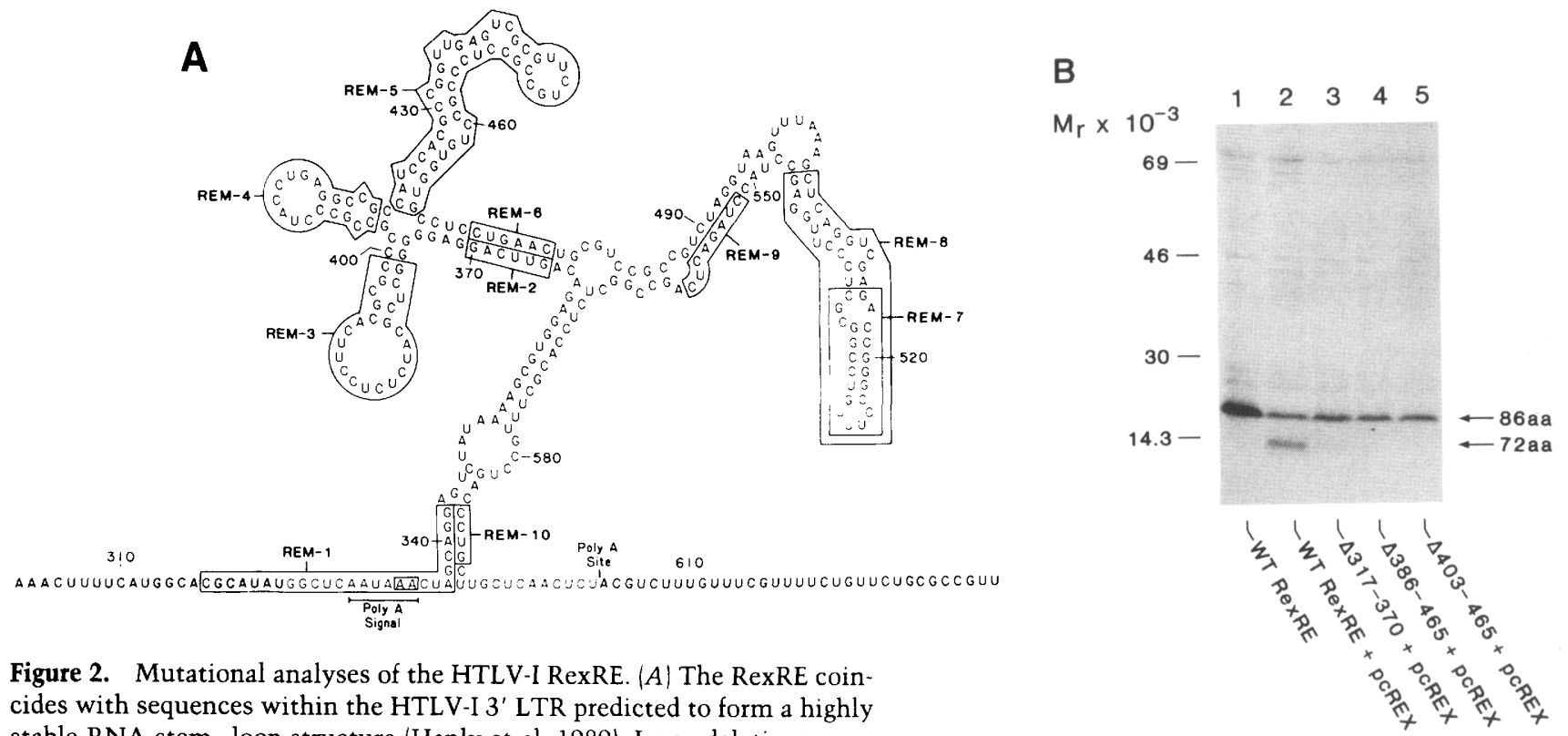

Figure 2. Mutational analyses of the HTLV-I RexRE. $|A|$ The RexRE coincides with sequences within the HTLV-I 3' LTR predicted to form a highly stable RNA stem-loop structure (Hanly et al. 1989). Large deletions were introduced into either the primary stem $(\Delta 317-370)$ or used to remove three $(\Delta 386-465)$ or two $(\Delta 403-465)$ of the stem-loop structures. Smaller deletion mutations were also introduced into the RexRE, as indicated schematically by REM 1-REM 10 . All of these mutations substitute the indicated boxed nucleotides with a BglII restriction site. All RexRE mutations retaining the HTLV-I polyadenylation signal (nucleotides 329-334) contain a 2-nucleotide point mutation $(\mathrm{AA} \rightarrow \mathrm{TT})$ within this hexamer motif at the boxed positions. Map position numbers (Seiki et al. 1983) corresponding to the identical nucleotides in the $5^{\prime}$ LTR are indicated. $(B$ and $C)$ Phenotypic analysis of the large and small RexRE deletion mutations. $(B)$ Mutations were analyzed after insertion of the mutated RexRE clones into the pgATAT expression vector. (C) The REM 1-REM 10 mutant clones of the RexRE (lanes 2-11), as well as the wild-type element (lane 1), were cotransfected into COS cells with an equal amount of pcREX. One-quarter of an alkaline lysis miniprep (Maniatis et al. 1982) of RexRE mutants $\Delta 317-370, \Delta 386-465$, and $\Delta 403-465$ was cotransfected with pcREX for the indicated precipitations. Labeled cell lysates were analyzed by immunoprecipitation with anti-Tat antibodies. Immunoprecipitated proteins were fractionated on $14 \%$ SDS-polyacrylamide gels; the migration of the 86 and 72-amino-acid forms of the Tat protein are indicated.

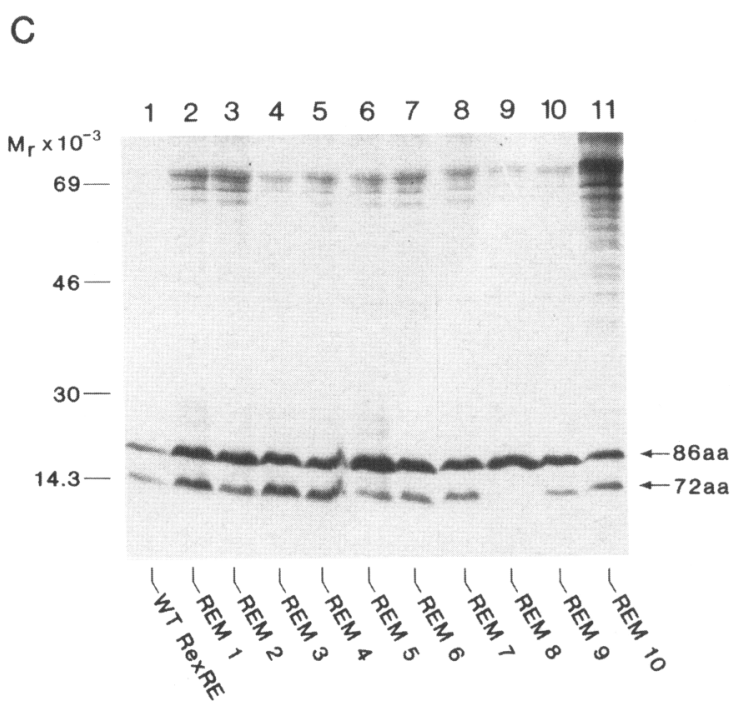

sponding to the REM 11, REM 12, and REM 13 mutations were replaced with a random sequence of identical length (see legend to Fig. 3). In addition, a double mutation incorporating both REM 12 and REM 13 was constructed. Though it alters all of the indicated nucleotides, this mutation creates a predicted stem structure at the subregion defined by the REM 12 and REM 13 mutations. Transfection studies revealed that alteration of the loop sequences corresponding to the REM 11 mutation had no effect on Rex responsiveness (Fig. 3B, lane 1). In contrast, the REM 12, REM 13, and the compensatory REM 12/13 mutations each produced near complete ablation of the Rex response (Fig. 3B, lanes 2-4). These findings emphasize the importance of the subregion defined by nucleotides 506-516 and 537-546 for Rex responsiveness. It is quite possible that this subregion forms a protein-binding site, either for Rex or a cellular factor. Notwithstanding, the action of this subregion is dependent on the generally intact secondary structure of the RexRE formed by the nucleotides located between 337 and 591.

\section{Different subregions of the HIV-1 RevRE are required for Rex and Rev action}

Several research groups have previously identified a cisacting element in the HIV-1 genome that is required for Rev responsiveness (RevRE) (Rosen et al. 1988; Dayton et al. 1989; Emerman et al. 1989; Felber et al. 1989; Hadzopoulou-Cladaras et al. 1989; Hammarskjold et al. 1989; Malim et al. 1989b; Zapp and Green 1989|. Like the RexRE, these sequences are predicted to form a highly ordered RNA stem-loop structure (Fig. 4A; Malim et al. 1989b). However, in contrast to the RexRE, the RevRE is only present in those singly spliced or unspliced viral mRNA species whose cytoplasmic appearance is regulated by Rev. We have shown previously that HTLV-I Rex can functionally substitute for the Rev pro- 


\section{Ahmed et al.}
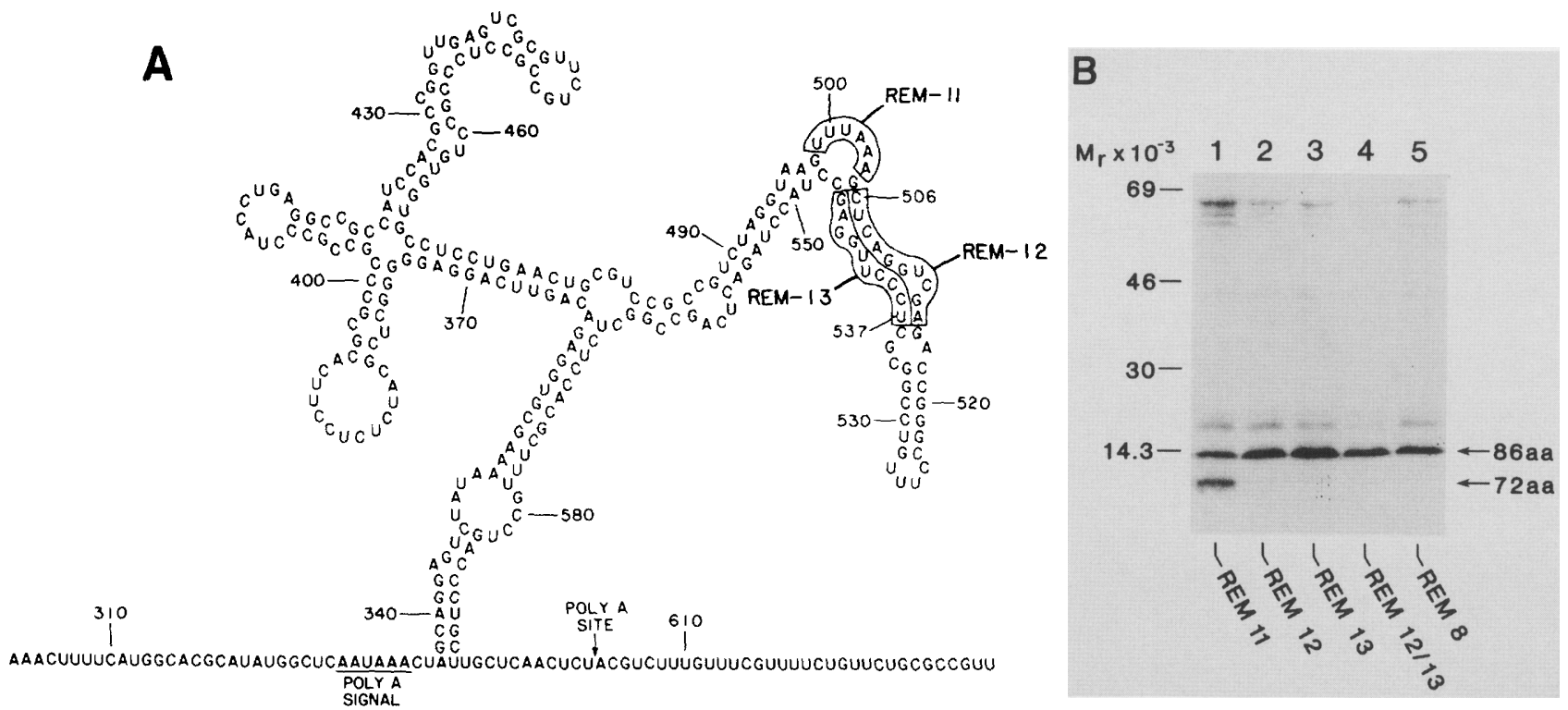

Figure 3. Fine mapping of the critical subregion of the HTLV-I RexRE. (A) Schematic summary of substitution mutations within the RexRE. The high lighted nucleotides were replaced with random sequences of equal length, as follows: REM 11 (UUUAAA $\rightarrow$ AGAUCU), REM 12 (CUCAGGUCGA $\rightarrow$ AGAUCUAUAU), REM 13 (UCCCUUGGAG $\rightarrow$ AUAUAGAUCU). REM 12/13 incorporates the changes of both the REM 12 and REM 13 mutations and is therefore predicted to create a perfect stem structure. $(B)$ Phenotypic analysis of substitution mutations. Mutations were subcloned into the pgDTAT reporter plasmid and assayed as described (see Fig. 2B).

A

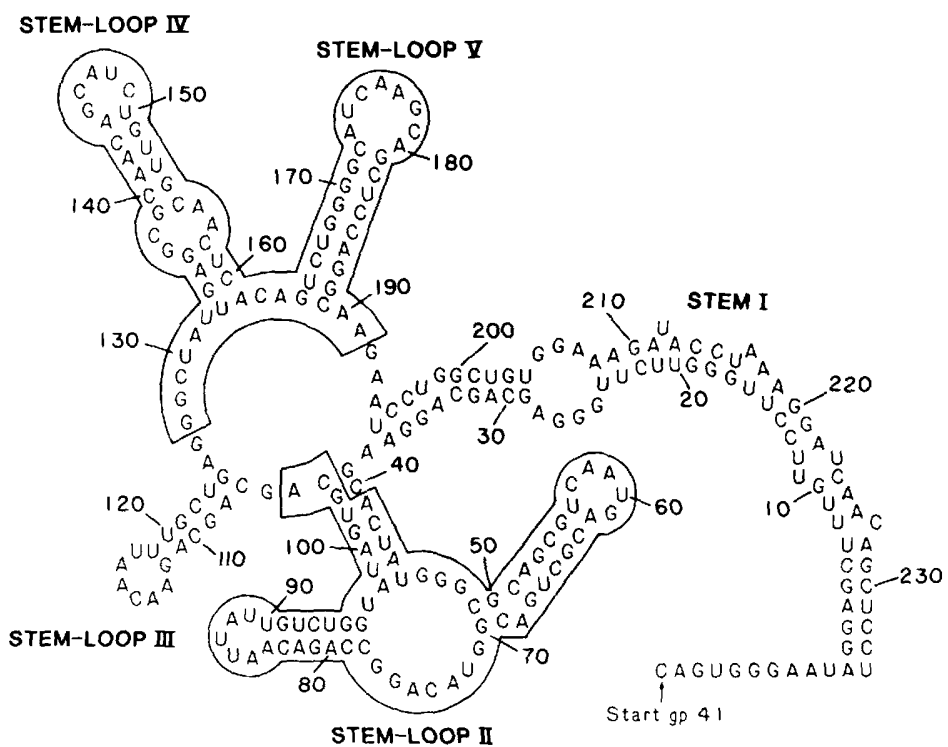

B

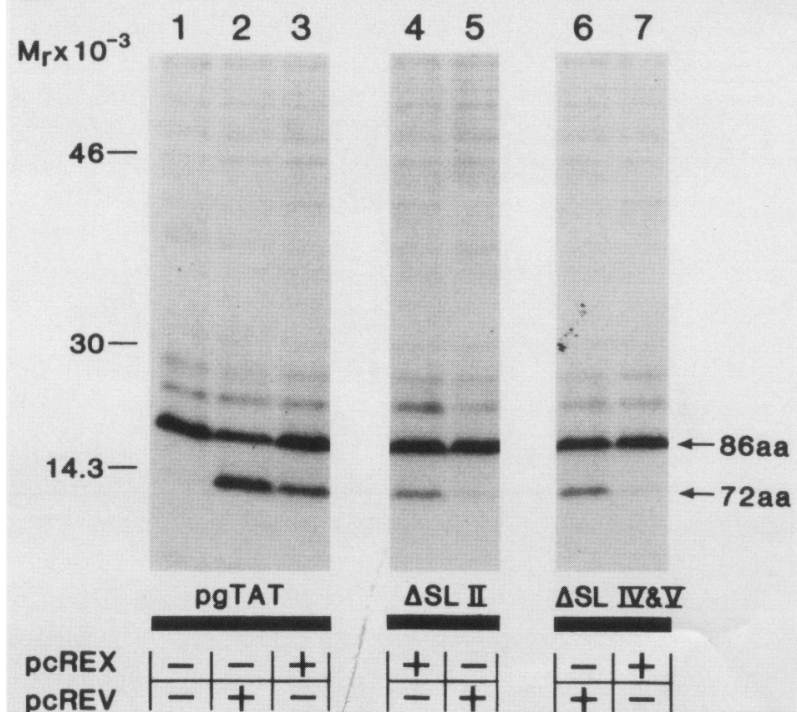

Figure 4. Comparison of Rex and Rev action through the HIV-l RevRE. (A) Schematic depiction of deletion mutations within the HIV-1 RevRE. The RevRE coincides with sequences within the HIV-1 env gene that are predicted to form a highly stable RNA stem-loop structure (Malim et al. 1989b). Two deletion mutations within the RevRE are depicted within the context of this structure ( $\Delta$ stem-loop II and $\Delta$ stem-loop IV and V). Both mutations substitute the indicated nucleotides with a SmaI restriction site. Map positions are indicated, and these mutants have been described previously (Malim et al. 1990). Stem-loop II is required for Rev action and contains a binding site for Rev. $(B)$ Phenotypic comparison of Rex and Rev function mediated through the mutated RevREs. COS cells were transfected with either the pcREX or pcREV expression vectors, as depicted by plus $(+\mid)$ signs, and an indicator plasmid containing either the full-length (pgTAT) or mutated $(\triangle S L$ II and $\triangle S L$ IV and V) RevRE at a $1: 4$ (wt/wt) ratio. Radiolabeled proteins from transfected cells were immunoprecipitated by anti-Tat antibodies and resolved on $14 \%$ SDS-polyacrylamide gels. The 86 - and 72-amino-acid forms of the Tat protein are indicated. 
tein of HIV-1 (Rimsky et al. 1988) and that this action of Rex is dependent on the presence of the RevRE (Hanly et al. 1989). Because the HTLV-I RexRE shares little identity with sequences in the RevRE, we compared Rex and Rev action on a series of RevRE deletion mutations to investigate whether the same or different subregions are required for Rex and Rev responsiveness. The HIV-1 expression vector used for this study, pgTAT (Malim et al. 1988), and its derivatives are similar to that used in the analysis of the RexRE, with the exception that either wild-type or mutant versions of the RevRE are present rather than the RexRE (Fig. 1B). In the presence of the wild-type RevRE, both Rex and Rev induced the expression of the truncated 72-amino-acid form of the Tat protein, reflecting translation of the unspliced vector RNA transcript (Fig. 4B, lanes 1-3). A series of deletions within the RevRE that either abolished Rev responsiveness or left it grossly intact (Malim et al. 1990) were then tested for their effects on Rex action (Fig. 4A). Of note, deletion of stem-loop II ( $\Delta$ SL II, deletion between nucleotides 41 and 105), which has been demonstrated to be critical for both Rev activity in vivo and its RNAbinding activity in vitro (Malim et al. 1990), failed to abolish the Rex response (Fig. 4B, lanes 4 and 5). Conversely, deletion of a distant subregion of the RevRE, encompassing stem-loops IV and V ( $\Delta$ SL IV and V, deletion between nucleotides 127 and 191) ablated Rex responsiveness but left Rev responsiveness largely intact (Fig. 4B, lanes 6 and 7). Deletion of sequences between these two subregions ( $\Delta 85$ and 117), had little effect on either Rex or Rev responsiveness (data not shown). A portion of the subregion of the HTLV-I RexRE that is crucial for $\operatorname{Rex}$ responsiveness (nucleotides 506-515) shares limited sequence identity with two regions within stem-loop $\mathrm{V}$ of the HIV-1 RevRE (nucleotides 158-169 and 180-193). However, deletion of stem-loop $\mathrm{V}$ alone had little effect on Rex action through the RevRE (data not shown). Thus, the critical sequences within the RevRE that are required for $\operatorname{Rex}$ responsiveness remain to be determined. Together, these results suggest that different subregions of the RevRE are crucial for Rex and Rev action and imply a possible difference in the binding sites for these trans-activators within this viral RNA regulatory element.

Rex and Rev function as dominant repressors following specific subregion deletion in the RevRE

Previous mutational analyses of the Rex and Rev proteins have revealed two distinct functional classes of mutations. One class of mutant proteins not only lacks biological activity but also is able to inhibit the action of the wild-type trans-activator. These proteins have been termed dominant negative or trans-dominant mutants. A second class of mutants corresponds to those that lack biological activity but fail to inhibit the action of the wild-type protein. This group is referred to as recessive negative mutants (Malim et al. 1989a; Rimsky et al. 1989). The identification of mutants falling into each of these two classes for Rex and Rev suggests the presence of at least two distinct functional domains within these proteins, namely a binding domain defined by the recessive negative mutations and an activation or effector domain defined by the trans-dominant mutations. Several precedents for such separable binding and activation domains exist, perhaps best illustrated by various transcription factors such as the yeast GAL4 protein (Ma and Ptashne 1987; Sadowski et al. 1988). The differing phenotypes of Rex and Rev, revealed by deletions within the RevRE coupled with the possibility of distinct functional domains in the trans-activators, prompted further analyses of function mediated through the RevRE. Specifically, to examine whether these domains in Rex and Rev might allow an interaction with another factor, we first tested whether an excess of Rev would alter the action of Rex by using the stem-loop II deletion of the RevRE (see Fig. 4A). Although it lacks the Rev-binding site, this mutant retains responsiveness to Rex (Fig. 5, lanes 6 and 7). When cotransfected at a fourfold excess, Rev (pcREV) was found to abolish Rex responsiveness mediated through the stem-loop II mutation (Fig. 5, lane 8). Thus, with this mutant RevRE, the wild-type Rev protein not only fails to function but also acts as a dominant repressor of Rex action. Analogously, Rex served as a dominant repressor of Rev when the stemloop IV and V deletion mutant of the RevRE (see Fig. 4A) was tested (Fig. 5, lane 11). This mutation abolishes Rex activity (Fig. 5, lane 10) but leaves Rev function largely intact (Fig. 5, lane 9). In contrast, little repression was observed when identical ratios of Rev and Rex were tested with the wild-type RevRE (Fig. 5, lanes 4 and 5). Together, these findings suggest that Rex and Rev may interact with a common cellular factor(s) that is both limiting and necessary for the action of these trans-activators. Alternatively, these viral proteins may form mixed heteromeric complexes that lack functional activity.

\section{Discussion}

Like all replication-competent retroviruses, HTLV-I must balance the production of spliced and unspliced forms of viral mRNA. Expression of the gag and env structural gene products, translated from an unspliced and singly spliced viral mRNA, respectively, requires both the trans-acting Rex protein and the RexRE (Inoue et al. 1987; Hidaka et al. 1988; Seiki et al. 1988; Hanly et al. 1989). The RexRE corresponds to a rather large RNA element comprised of 255 bases that is predicted to form a remarkably ordered stem-loop structure (Hanly et al. 1989). Consistent with the importance of this RNA structure for Rex action, large internal deletions within the RexRE that drastically alter its predicted stability also destroy Rex responsiveness. In contrast, with one exception, smaller deletions that remove discrete stemloop or stem subregions have little or no effect on Rex function. The one intriguing exception involves the deletion or substitution of nucleotides between 506 and 515 and between 537 and 546 which, together, are predicted to form a weak stem structure (see Fig. 2A). We hypothesize that this critical subregion may serve as a 


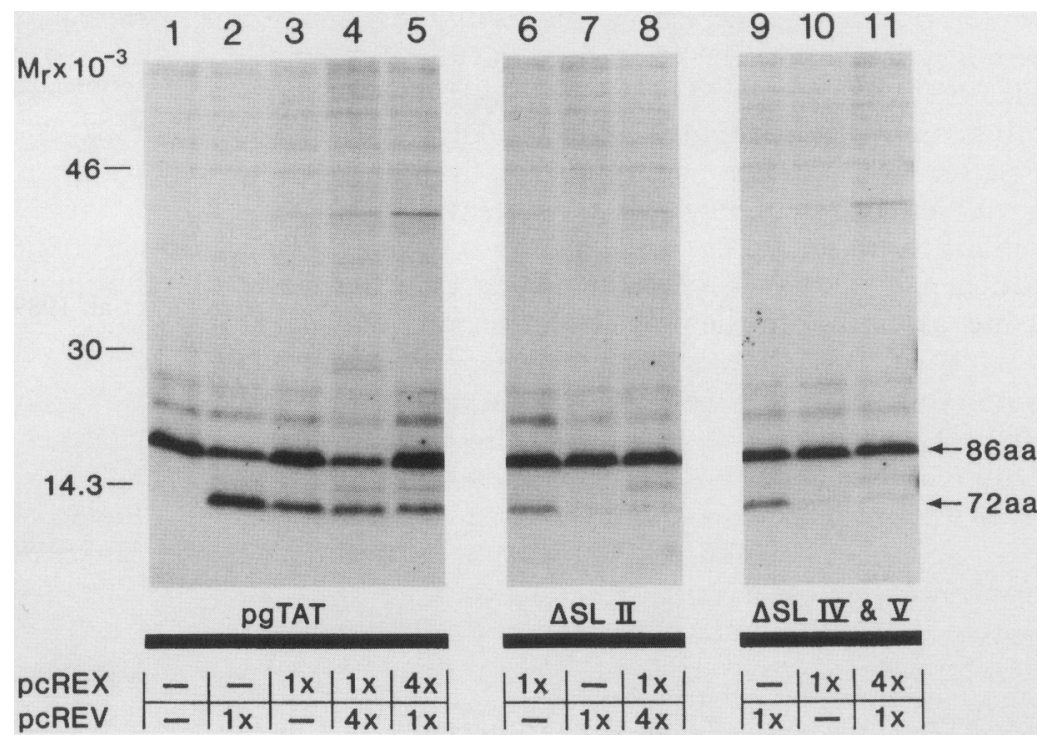

Figure 5. Competitive inhibition by Rex and Rev is revealed by deletions in the RevRE. COS cells were transfected with either pcREX and/or pcREV at the indicated ratios. Each transfection also included an indicator vector containing either the full-length (pgTAT) or mutated ( $\triangle$ SL II and $\triangle$ SL IV and V; Fig. 3A) RevRE, as indicated. Plasmid DNA concentration was held constant in all transfections by the inclusion of $\mathrm{pBC} / \mathrm{CMV} / \mathrm{IL}-2$ control DNA. Radiolabeled proteins from the transfected cells were immunoprecipitated with the anti-Tat antibodies. The 86- and 72-amino-acid forms of the Tat protein are indicated.

protein-binding site in the RexRE, either for Rex or for a cellular factor with affinity for RNA. The inability of the compensatory REM 12/13 double mutation to restore Rex responsiveness suggests that both the actual sequence of nucleotides and the structure formed by this sequence are important in the Rex response. This is not altogether surprising, as sequence and secondary structure are inextricably linked in RNA. The functional importance of this subregion is strengthened further by the sequence similarity of its $5^{\prime}$ portion with a subregion in the putative RNA response elements of both HTLV-II (Kim et al., in prep.) (11 of 12 nucleotides) and the bovine leukemia virus (Derse 1988) (9 of 12 nucleotides). These viruses are related but distinct members of the HTLV family.

The predicted RNA secondary structure formed by the RexRE has also been suggested to play a role in polyadenylation of viral transcripts (Seiki et al. 1983), as its formation approximates the distant polyadenylation hexamer motif (AAUAAA) with ultimate site of $3^{\prime}$ cleavage (for review, see Birnstiel et al. 1985). In this regard, the extraordinarily long $\mathrm{R}$ regions within the LTRs of both HTLV-II and bovine leukemia virus (BLV) also form predicted stem-loop structures, a finding that distinguishes this family of retroviruses from all others (Sagata et al. 1984; Kim et al., unpubl.). Although Rex responsiveness and polyadenylation can be clearly segregated (Hanly et al. 1989), we have found that deletion of the major stem within the RexRE abolishes not only Rex responsiveness but also polyadenylation (Y. Ahmed, G. Gilmartin, S. Hanly, J. Nevins, and W. Greene, unpubl.). Thus, this RNA secondary structure appears to mediate two discrete functions, namely Rex responsiveness and polyadenylation. This latter action may explain why the RexRE has been evolutionarily preserved in the multiply spliced 2.0-kb class of viral mRNAs. Although cytoplasmic expression of these completely spliced mRNAs occurs independent of Rex, their stability is likely dependent on effective polyadenylation.
A related but distinct RNA response element, termed the RevRE, is present in HIV-1 and is similarly required for expression of the viral structural genes (Rosen et al 1988; Emerman et al. 1989; Hadzopoulou-Cladaras et al. 1989; Hammarskjold et al. 1989; Malim et al. 1989b). The HIV-1 RevRE is located in the env gene and contains a binding site for the Rev protein (Daly et al. 1989; Zapp et al. 1989; Malim et al. 1990). Mutational analysis of the RevRE, combined with in vivo assays of Rev function, have allowed identification of a subregion of the RevRE that is essential for biological activity (Dayton et al. 1989; Malim et al. 1990). Of note, this subregion corresponds precisely to the sequences required for Rev binding to the RevRE (Malim et al. 1990). In this regard, our finding that significant differences exist in the subregions of the RevRE required for Rex and Rev responsiveness raises the possibility that corresponding differences may exist in the protein-binding sites that mediate Rex and Rev action through the RevRE.

Although it is known that both Rev and Rex allow the cytoplasmic appearance of incompletely spliced viral mRNAs encoding the structural proteins (Hidaka et al. 1988; Malim et al. 1988; Hanly et al. 1989), the exact mechanism by which these trans-activators achieve this effect remains unclear. Actions at the level of mRNA splicing, nuclear transport, and RNA stabilization and translation have been proposed (Feinberg et al. 1986; Sodroski et al. 1986; Chang and Sharp 1989; Felber et al. 1989; Hanly et al. 1989; Malim et al. 1989b). We were interested in investigating whether the action of Rex and Rev may involve a direct physical interaction, either between the trans-activators or with a common cellular intermediate. Using deletion mutations within the RevRE that abolish the action of one but not the other trans-activator, we now demonstrate that the inactive trans-activator functions as a dominant repressor of its active counterpart. Thus, depending on the RevRE deletion mutant, Rex can inhibit Rev action or Rev can abrogate Rex activity. As mentioned, this phenomenon has 
several potential explanations, including the formation of mixed Rex-Rev oligomers, the existence of multiple inactive binding sites within the RevRE for both proteins, or a squelching mechanism (for review, see Ptashne 1988) involving the interaction of Rex and Rev with a titratable cellular factor(s). Given the post-transcriptional action of Rex and Rev, that this latter interaction may involve a cellular factor active in spliceosome assembly or the nucleocytoplasmic transfer of viral RNA. Identification of such a putative factor would help elucidate the precise biochemical basis for Rex and Rev action. Finally, our findings further emphasize the multiple molecular mechanisms by which dominant negative repressors may potentially act.

\section{Methods}

Construction of expression vectors

The RexRE mutants (REM 1-REM 13, $4317-370, \Delta 386-465$, $\Delta 403-465$ ), were derived from a RexRE fragment (nucleotides 302-635) (Seiki et al. 1983) that was subcloned from the HTLV-I LTR by use of the polymerase chain reaction (PCR; Hanly et al. 1989). The reaction included a coding-strand synthetic oligonucleotide that incorporated a 2 -bp point mutation within the polyadenylation signal at nucleotides 333-334 (AATAAA $\rightarrow$ AATATT) that abolishes polyadenylation at this site (Y. Ahmed, G. Gilmartin, S. Hanly, J. Nevins, and W. Greene, unpubl.). The primers also inserted an $\mathrm{XbaI}$ restriction site $\left(5^{\prime}\right.$ TCTAGA-3') at both ends of the fragment to facilitate subcloning. Oligonucleotide-directed mutagenesis (Taylor et al. 1985) of this fragment was performed in the M13 bacteriophage (Amersham, Arlington Heights, IL). All mutations within the RexRE introduced a unique BglII site $\left(5^{\prime}\right.$-AGATCT- $\left.3^{\prime}\right)$; each mutation is illustrated in Figure 2A. The mutated RexREs were inserted into the HIV expression vector pg $\Delta \mathrm{TAT}$ (Malim et al. $1989 \mathrm{~b}$ ) at the unique $\mathrm{XbaI}$ site to create the series of $\mathrm{pg} \Delta \mathrm{TAT}$ REM 1-REM 13 constructs, as well as the $\Delta 317-370$, $\Delta 386-465$, and $\Delta 403-465$ mutants. The REM l-REM $13 \mathrm{mu}-$ tations were verified by DNA sequencing. The RevRE mutant plasmids ( $\Delta$ stem-loop II and $\Delta$ stem-loop IV and V) were similarly derived by using oligonucleotide-directed mutagenesis (Malim et al. 1990).

The pgTAX-R' construct was derived form the HTLV-I pgTAX vector (Hanly et al. 1989) by blunt-ended insertion of the RexRE PCR fragment containing a wild-type polyadenylation signal at the unique 3' BstEII restriction site. The construction of pgTAT and its derivative pg $\triangle T A T$, as well as pcREV, pcREX, and pBC12/CMV/IL-2, has been described /Cullen 1986; Malim et al. 1988, 1989b; Rimsky et al. 1988).

\section{Cell culture and transfection}

Monkey kidney COS cells were maintained and transfected by using DEAE-dextran and chloroquine as described previously (Cullen 1986, 1987). The concentration of DNA in all transfections was held constant at $2.5 \mu \mathrm{g} / \mathrm{ml}$ by inclusion of the $\mathrm{pBC12/CMV/IL-2} \mathrm{control} \mathrm{plasmid} \mathrm{as} \mathrm{needed.}$

\section{Immunoprecipitation analysis}

Functional analyses of the RexRE constructs in the indicator vectors pgTAX-R' and $\mathrm{pg} \Delta T A T / R^{\prime}$ and the RevRE constructs in pgTAT were performed by immunoprecipitation of $\left[{ }^{35} S \mid c y s-\right.$ teine-labeled Tax, Env, and Tat proteins, $48 \mathrm{hr}$ after transfection (Hanly et al. 1989). The rabbit polyclonal anti-Tat and anti-Tax antisera, as well as the human anti-Env monoclonal antibody, $0.5 \alpha$, were described previously (Matsushita et al. 1986; Hauber et al. 1987; Rimsky et al. 1988). Immunoprecipitations were analyzed by SDS-PAGE and visualized by autoradiography.

\section{Computer analysis}

The derivation of the secondary structure proposed for both the RexRE and the RevRE has been described (Le et al. 1988; Hanly et al. 1989; Malim et al. 1989 b).

\section{Acknowledgments}

We thank Dr. H. Mitsuya for the gift of the $0.5 \alpha$ monoclonal antibody specific for HTLV-I Env, and Ms. Bonnie Kissell for secretarial assistance in the preparation of this manuscript. Y.F.A. is a Howard Hughes Medical Institute Medical Student Research Training Fellow.

\section{References}

Birnstiel, M.L., M. Busslinger, and K. Strub. 1985. Transcription termination and $3^{\prime}$ processing: The end is in site! Cell 41: 349-359.

Chang, D.D. and P.A. Sharp. 1989. Regulation by HIV Rev depends upon recognition of splice sites. Cell 59: 789-795.

Cullen, B.R. 1986. Trans-activation of human immunodeficiency virus occurs via a bimodal mechanism. Cell 46: $973-$ 982.

1987. Use of eukaryotic expression technology in the functional analysis of cloned genes. Methods Enzymol. 152: $684-704$

Cullen, B.R. and W.C. Greene. 1989. Regulatory pathways governing HIV-1 replication. Cell 58: 423-426.

Daly, T.J., K.S. Cook, G.S. Gray, T.E. Maione, and J.R. Rusche. 1989. HIV-1 recombinant Rev protein binds specifically to the Rev response element in vitro. Nature 342: 816-819.

Dayton, E.T., D.M. Powell, and A.I. Dayton. 1989. Functional analysis of CAR, the target sequence for the Rev protein of HIV-1. Science 246: 1625-1628.

Derse, D. 1988. Trans-acting regulation of bovine leukemia virus mRNA processing. J. Virol. 62: 1115-1119.

Emerman, M., R. Vazeux, and K. Peden. 1989. The rev gene product of the human immunodeficiency virus affects envelope-specific RNA localization. Cell 57: 1155-1165.

Feinberg, M.B., R.F. Jarrett, A. Aldovini, R.C. Gallo, and F. Wong-Staal. 1986. HTLV-III expression and production involve complex regulation at the levels of splicing and translation of viral RNA. Cell 46: 807-817.

Felber, B.K., M. Hadzopoulou-Cladaras, C. Cladaras, T. Copeland, and G.N. Pavlakis. 1989. Rev protein of human immunodeficiency virus type $l$ affects stability and transport of the viral mRNA. Proc. Natl. Acad. Sci. 86: 1495-1499.

Hadzopoulou-Cladaras, M., B.K. Felber, C. Cladaras, A. Athanassopoulos, A. Tse, and G.N. Pavlakis. 1989. The rev (trs/ art) protein of human immunodeficiency virus type 1 affects viral mRNA and protein expression via a cis-acting sequence in the env gene product. I. Virol. 63: 1265-1274.

Hammarskjold, M.-L., J. Heimer, B. Hammarskjold, I. Sangwan, L. Albert, and D. Rekosh. 1989. Regulation of human immunodeficiency virus env gene product. I. Virol. 63: 19591966.

Hanly, S.M., L.T. Rimsky, M.H. Malim, J.H. Hauber, M. Duc Dodon, S.-Y. Le, J.V. Maizel, B.R. Cullen, and W.C. Greene. 1989. Comparative analysis of the HTLV-I Rev and HIV-1 Rev trans-regulatory proteins and their RNA response elements. Genes Dev. 3: 1534-1544. 
Hauber, J., A. Perkins, E.P. Heimer, and B.R. Cullen. 1987. Trans-activation of human immunodeficiency virus gene expression is mediated by nuclear events. Proc. Natl. Acad. Sci. 84: 6364-6368.

Hidaka, M., J. Inoue, M. Yoshida, and M. Seiki. 1988. Post-transcriptional regulator (rex) of HTLV-I initiates expression of viral structure proteins but suppresses expression of regulatory proteins. $E M B O$ J. 7: 519-523.

Inoue, J.-I., M. Yoshida, and M. Seiki. 1987. Transcriptional $\left(\mathrm{p}^{4} 0^{\mathrm{x}}\right)$ and post-transcriptional $\left(\mathrm{p} 27^{\mathrm{x}}\right.$-III) regulators are required for the expression and replication of human $\mathrm{T}$-cell leukemia virus type I genes. Proc. Natl. Acad. Sci. 84: 3653-3657.

Le, S.-Y., J.-H. Chen, M.J. Braun, M.A. Gonda, and J.V. Maizel. 1988. Stability of RNA stem-loop structure and distribution of non-random structure in the human immunodeficiency virus (HIV-1). Nucleic Acids Res. 16: 5153-5168.

Ma, J. and M. Ptashne. 1987. The carboxy-terminal 30 amino acids of GAL4 are recognized by GAL80. Cell 50: 137-142.

Malim, M.H., J. Hauber, R. Fenrick, and B.R. Cullen. 1988. Immunodeficiency virus rev trans-activator modulates the activity of the viral regulatory genes. Nature 335: 181-183.

Malim, M.H., S. Bohnlein, J. Hauber, and B.R. Cullen. 1989a. Functional dissection of the HIV-1 Rev trans-activatorderivation of a trans-dominant repressor of Rev function. Cell 58: 205-214.

Malim, M.H., J. Hauber, S.-Y Le, J.V. Maizel, and B.R. Cullen. 1989b. The HIV-1 rev trans-activator acts through a structured target sequence to activate nuclear export of unspliced viral mRNA. Nature 338: 254-257.

Malim, M.H., L.S. Tiley, D.F. McCarn, J.R. Rusche, J. Hauber, and B.R. Cullen. 1990. HIV-1 structural gene expression requires binding of the Rev trans-activator to its RNA target sequence. Cell 60: 675-683.

Maniatis, T., E.F. Fritsch, and J. Sambrook. 1982. Molecular cloning: A laboratory manual. Cold Spring Harbor Laboratory Press, Cold Spring Harbor, New York.

Matsushita, S., M. Robert-Guroff, J. Trepel, J. Cossman, H. Mitsuya, and S. Broder. 1986. Human monoclonal antibody directed against an envelope glycoprotein of human T-cell leukemia virus type I. Proc. Natl. Acad. Sci. 83: 2672-2676.

Ptashne, M. 1988. How eukaryotic transcriptional activators work. Nature 335: 683-689.

Rimsky, L.T., J. Hauber, M. Dukovich, M.H. Malim, A. Langlois, B.R. Cullen, and W.C. Greene. 1988. Functional replacement of the HIV-1 Rev protein by the HTLV-I Rex protein. Nature 335: 738-740.

Rimsky, L.T., M. Duc Dodon, E.P. Dixon, and W.C. Greene. 1989. Mutational analysis of the HTLV-I Rex transactivator: Transdominant inactivation of HTLV-I and HIV-1 gene expression. Nature 341: 453-456.

Rosen, C.A., E. Terwilliger, A. Dayton, J.G. Sodroski, and W.A. Haseltine. 1988. Intragenic cis-acting art gene responsive sequences of the human immunodeficiency virus. Proc. Natl. Acad. Sci. 85: 2071-2075.

Sadaie, M.R., T. Benter, and F. Wong-Staal. 1988. Site-directed mutagenesis of two trans-regulatory genes (tat-III, trs) of HIV-1. Science 239: 910-914.

Sadowski, I., J. Ma, S. Triezenberg, and M. Ptashne. 1988. Gal4VP16 is an unusually potent transcriptional activator. $\mathrm{Na}$ ture 335: 563-564.

Sagata, N., T. Yasunaga, Y. Ogawa, J. Tsuzuku-Kawamura, and Y. Ikawa. 1984. Bovine leukemia virus: Unique structural features of its long terminal repeats and its evolutionary relationship to human T-cell leukemia virus. Proc. Natl. Acad. Sci. 81: 4741-4745.
Seiki, M., S. Hattori, and M. Yoshida. 1982. Human adult T-cell leukemia virus: Molecular cloning of the provirus DNA and unique terminal structure. Proc. Natl. Acad. Sci. 79: 68997902.

Seiki, M., S. Hattori, Y. Hirayama, and M. Yoshida. 1983 Human adult T-cell leukemia virus: Complete nucleotide sequence of the provirus genome integrated in leukemia cell DNA. Proc. Natl. Acad. Sci. 80: 3618-3622.

Seiki, M., A. Hikikoshi, T. Taniguchi, and M. Yoshida. 1985. Expression of the pX gene of HTLV-I: General splicing mechanism in the HTLV family. Science 227: 1227-1229.

Seiki, M., J.-I. Inoue, M. Hidaka, and M. Yoshida. 1988. Two cis-acting elements responsible for post-transcriptional trans-regulation of gene expression of human T-cell leukemia virus type I. Proc. Natl. Acad. Sci. 85: 7124-7128.

Sodroski, J., W.C. Goh, C. Rosen, A. Dayton, E. Terwilliger, and W. Haseltine. 1986. A second post-transcriptional trans-activator gene required for HTLV-III replication. Nature 321: $412-417$

Taylor, J.W., J. Ott, and F. Eckstein. 1985. The rapid generation of oligonucleotide-directed mutations at high frequency using phosphorothioate-modified DNA. Nucleic Acids Res. 13: $8765-8785$.

Terwilliger, E., R. Burghoff, R. Sia, J. Sodroski, W. Haseltine, and C. Rosen. 1988. The art gene product of human immunodeficiency virus is required for replication. I. Virol. 62: 655-658.

Yoshida, M. and M. Seiki. 1987. Recent advances in the molecular biology of HTLV-I: Trans-activation of viral and cellular genes. Annu. Rev. Immunol. 5: 541-559.

Zapp, M.L. and M.R. Green. 1989. Sequence-specific RNA binding by the HIV-1 Rev protein. Nature 342: 714-716. 


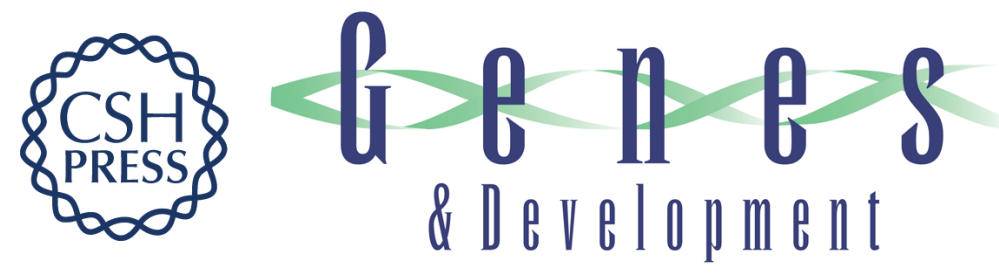

\section{Structure-function analyses of the HTLV-I Rex and HIV-1 Rev RNA response elements: insights into the mechanism of Rex and Rev action.}

Y F Ahmed, S M Hanly, M H Malim, et al.

Genes Dev. 1990, 4:

Access the most recent version at doi:10.1101/gad.4.6.1014

References This article cites 40 articles, 17 of which can be accessed free at: http://genesdev.cshlp.org/content/4/6/1014.full.html\#ref-list-1

License

Email Alerting Service

Receive free email alerts when new articles cite this article - sign up in the box at the top right corner of the article or click here.

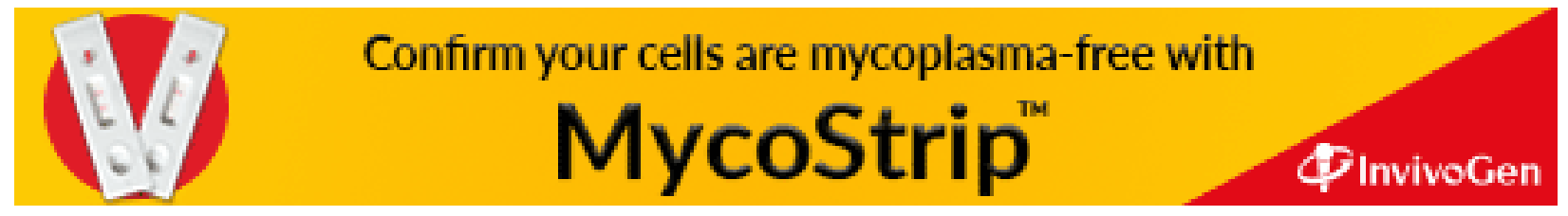

